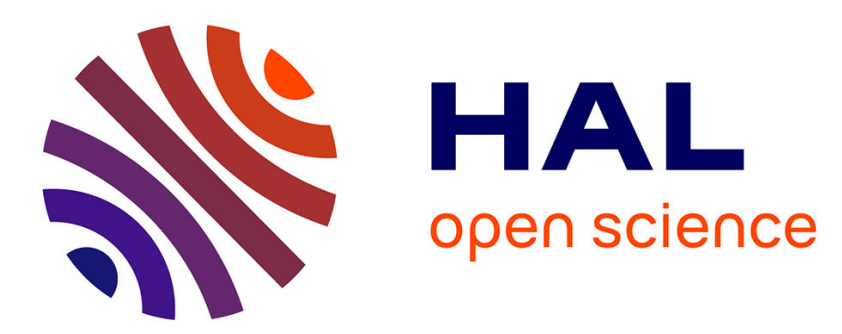

\title{
Critical attenuation of sound in liquid-helium films at 9 $\mathrm{GHz}$
}

\author{
J. Joffrin, B. Lambert, D. Salin
}

\section{To cite this version:}

J. Joffrin, B. Lambert, D. Salin. Critical attenuation of sound in liquid-helium films at $9 \mathrm{GHz}$. Journal de Physique Lettres, 1976, 37 (10), pp.255-259. 10.1051/jphyslet:019760037010025500 jpa00231287

\section{HAL Id: jpa-00231287 https://hal.science/jpa-00231287}

Submitted on 1 Jan 1976

HAL is a multi-disciplinary open access archive for the deposit and dissemination of scientific research documents, whether they are published or not. The documents may come from teaching and research institutions in France or abroad, or from public or private research centers.
L'archive ouverte pluridisciplinaire HAL, est destinée au dépôt et à la diffusion de documents scientifiques de niveau recherche, publiés ou non, émanant des établissements d'enseignement et de recherche français ou étrangers, des laboratoires publics ou privés. 


\author{
Classification \\ Physics Abstracts \\ $7.250-7.270-7.720-7.880$
}

\title{
CRITICAL ATTENUATION OF SOUND IN LIQUID-HELIUM FILMS AT 9 GHz
}

\author{
J. JOFFRIN, B. LAMBERT and D. SALIN \\ Laboratoire d'Ultrasons (*), Université Pierre-et-Marie-Curie, \\ Tour 13, 4 place Jussieu, 75230 Paris Cedex 05, France
}

(Reçu le 9 avril 1976, révisé le 18 juin 1976, accepté le 23 juin 1976)

\begin{abstract}
Résumé. - Nous avons mesuré l'atténuation critique ultrasonore à $9 \mathrm{GHz}$ dans des films d'hélium superfluide. On interprète l'exposant critique $0,65 \pm 0,15$ de la divergence de l'atténuation dans l'hélium liquide en faisant appel aux lois d'échelles dynamiques. La température d'apparition de la suprafluidité dépend de l'épaisseur du film; cet effet est expliqué en utilisant les conditions de Ginzburg-Pitaevsky.
\end{abstract}

Abstract. - We have measured the critical ultrasonic attenuation in superfluid helium films at $9 \mathrm{GHz}$. The critical exponent $0.65 \pm 0.15$ of the divergence of the attenuation in liquid helium is interpreted with dynamical scaling arguments. The temperature marking the onset of superfluidity depends on the film thickness; this effect is explained in terms of the Ginzburg-Pitaevsky conditions.

Most of the singularities of the transport coefficients near the $\lambda$ transition of helium have been analysed within the framework of the dynamical scaling method by considering processes in which one transport mode breaks up into several of the same nature. As a consequence, both the second sound damping and the thermal conductivity $K$ have been shown to diverge like $\varepsilon^{-1 / 3}$ [1] where

$$
\varepsilon=\frac{T_{\lambda}-T}{T_{\lambda}}
$$

On the contrary, the first sound damping is generally thought to be due to the coupling of the density with the order parameter; two effects are observable :

1) Around $T_{\lambda}$, the fluctuations of the order parameter induce a divergence of the attenuation which in the hydrodynamic regime is described adequately by

$$
\alpha \simeq \frac{\Delta c_{1,2}}{c_{1}^{2}} \frac{\omega^{2} \tau_{2}}{1+\omega^{2} \tau_{2}^{2}}
$$

where $\tau_{2} \simeq \xi / c_{2}$ is the characteristic relaxation time of the order parameter (second sound velocity $c_{2} \sim \varepsilon^{1 / 3}$, coherence length $\xi=\xi_{0} \varepsilon^{-2 / 3} ; \tau_{2}$ varies like $\left.\varepsilon^{-1}\right) ; \Delta c_{1,2}$ is a constant related to the coupling of first and second sound [2]. Thus the divergence of $\alpha$ is proportional to $\varepsilon^{-1}$ as long as $\omega \tau_{2} \ll 1$ [3]. In the

$\left(^{*}\right)$ Associated with the Centre National de la Recherche Scientifique. opposite limit $\left(\omega \tau_{2} \gg 1\right)$, the attenuation which is no longer represented by (1), saturates and becomes independent of the frequency.

2) In the low temperature phase, a second additional mechanism takes place. The relaxation of the order parameter leads to an attenuation having the same behaviour as (1) which peaks below $T_{\lambda}$ at a temperature given by

$$
\omega \tau_{2}=1 \text {. }
$$

Experimentally the condition (2) has been carefully explored by Williams and Rudnick [3]. They have shown that (2) is verified at $1 \mathrm{MHz}$ for

$$
T_{\lambda}-T=7 \times 10^{-5} \mathrm{~K} \text {; }
$$

this would lead, at $9 \mathrm{GHz}$, to a peak at

$$
\Delta T=T_{\lambda}-T=0.7 \mathrm{~K}
$$

very far from $T_{\lambda}$ with a value of $3000 \mathrm{~cm}^{-1}$.

Most of the former experiments on attenuation or on the velocity of acoustical waves near $T_{\lambda}$ have been performed in the low frequency range $(20 \mathrm{kHz}$ to $10 \mathrm{MHz}$ ). The experimental results are well understood within the frame work of (1) and have been reviewed in different issues [4].

However, some experiments show that at much higher frequencies, ranging from $1 \mathrm{GHz}$ [5] to $9 \mathrm{GHz}$ [6], a strong divergence persists around $T_{\lambda}$ despite the fact that $\omega \tau_{2} \gg 1$; this is clearly incompatible with the picture which leads to (1) and to 
the value given in (3). Thus, such high frequencies would require another explanation.

In this paper we report new quantitative results on the divergence of the sound absorption obtained in the high frequency regime in the superfluid phase and we suggest an explanation in terms of dynamical scaling and which corresponds to a homogeneous decay of one acoustical mode into two phonons modes.

The technique used to work at such a high frequency has been previously described [6]; the modifications introduced here permit the growth of thicker films and allow only a very weak flow of gas to enter the cell. The resonance lines therefore have a correct amplitude and the attenuation coefficient $k^{\prime \prime}$ or $\alpha$ is not overestimated. These are the decisive improvements in comparison with [6] in the vicinity of $T_{\lambda}$. In this new apparatus, the saturated film thickness is estimated to be $300 \AA$ and consequently, several mechanical resonances of the film are observed when

$$
d=(2 n+1) \frac{\lambda}{4}
$$

( $d$ film thickness, $\lambda$ acoustical wavelength, $n$ integer); at $9 \mathrm{GHz}, \lambda / 4 \simeq 60 \AA \simeq 17$ atomic layers. Figure 1 shows a typical experimental record of the acoustical signal observed by reflexion on the $20^{\text {th }}$ echo as a function of $\Delta P=P-P_{0}\left(T_{0}\right)$ where $P$ is the pressure in the cell and $P_{0}\left(T_{0}\right)$ is the saturated vapor pressure of liquid helium at temperature $T_{\lambda} . \Delta P$ and $d$ are related by

$$
k_{\mathrm{B}} T_{0} \frac{\Delta P}{P_{0}}=\frac{\alpha}{d^{3}}
$$

where $\alpha$ is the Van der Waals coefficient.

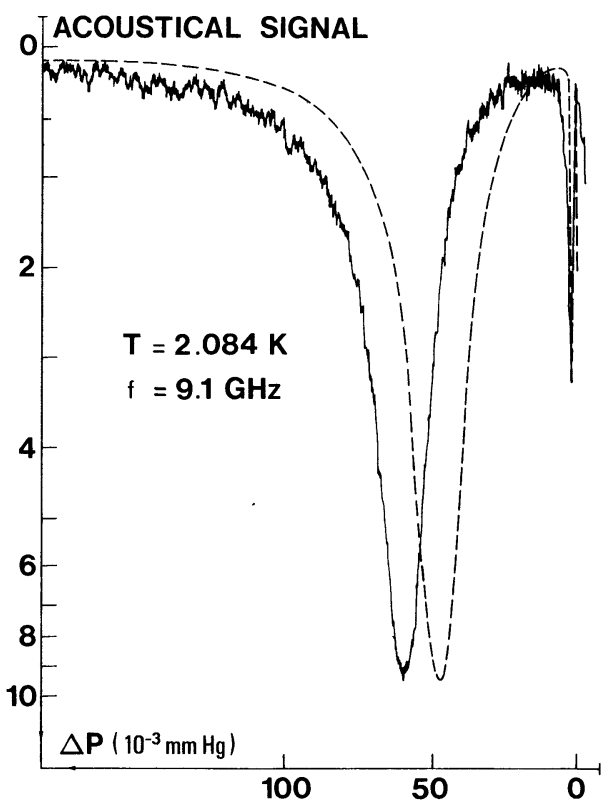

Fig. 1. - Experimental record of the acoustical signal for the $20^{\text {th }}$ echo versus the pressure $\Delta P$. The main resonance line corresponds to the first resonance $(\lambda / 4)$, the small one to the second $(3 \lambda / 4)$. The dashed line curve is computed (see text).
The dashed curve of figure 1 is computed using formula (4) and expression (1) of reference [6]; the origin $(\Delta P=0)$ of both curves is the same; the pressure difference of the first resonance line between the experimental and calculated curves is due to the solid layer at the interface substrate-film [7].

From the intensity of each resonance one can compute, using formula (1) of reference [6], a value of the ultrasonic attenuation $k^{\prime \prime}$. (Close to $T_{\lambda}$ we take into account the small variation of the first sound velocity $c_{1}[8]$.)

The temperature dependence of the ultrasonic attenuation $k^{\prime \prime}$ for the first resonance $\left(d_{1}=\lambda / 4\right)$ and for the second one $\left(d_{2}=3 \lambda / 4\right)$ is plotted on figure 2 ; several features must be noticed :

- The corresponding values $k_{1}^{\prime \prime}$ and $k_{2}^{\prime \prime}$ measured from the resonance lines remain identical when the temperature is increased until a certain value $T_{1}$; above $T_{1}, k_{1}^{\prime \prime}$ remains constant while $k_{2}^{\prime \prime}$ further increases $\left(\Delta T_{1}=T_{\lambda}-T_{1}=45 \mathrm{mK}\right)$; in the same way the divergence of $k_{2}^{\prime \prime}$ presents a saturation for

$$
\Delta T<\Delta T_{2} \simeq 5 \mathrm{mK} \text {. }
$$

- We have plotted the measurements obtained by $\mathrm{P}$. Berberich et al. [9] by Brillouin diffraction at $705 \mathrm{MHz}$ on the same graph (Fig. 2). Their values of $k^{\prime \prime}$ were extended to take into account the expected square frequency dependence of the attenuation.

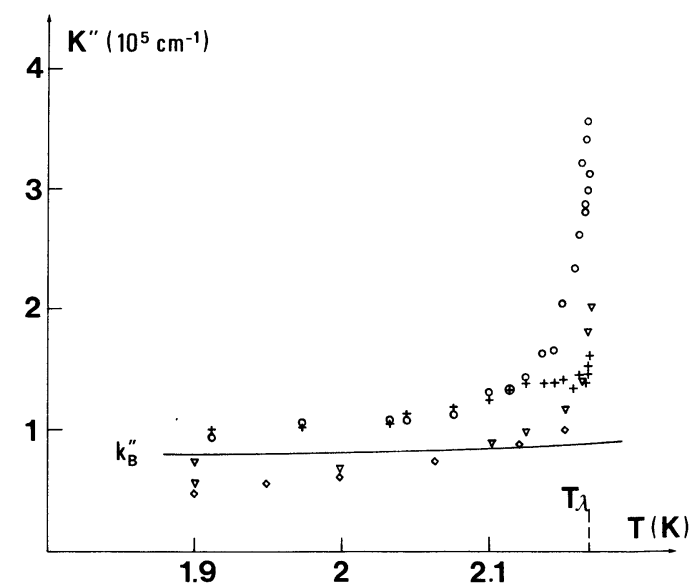

Fig. 2. - Temperature dependence of the ultrasonic attenuation in liquid helium film. The $(+)$ and $(0)$ correspond respectively to values of $k^{\prime \prime}$ given by the first and second resonance. Assuming a square frequency dependence $(\nabla)$ and $(\diamond)$ are the results of [5] and of [9]. The curve $k_{\mathrm{B}}^{\prime \prime}$ is our temperature dependent background.

- The values of $k^{\prime \prime}$ obtained by [5] in a more difficult experiment at $1 \mathrm{GHz}$ are extrapolated in the same way.

A comparison of these two results with ours shows that the square frequency dependence of the ultrasonic attenuation is not exactly fullfilled. Our values are $30 \%$ larger at $2 \mathrm{~K}$. This discrepancy is not too severe if we recall that the results are extrapolated 
from low frequencies where the attenuation is not described by the same processes as we will see below.

In order to extract the critical behaviour of $k^{\prime \prime}$ from our measurements we give in figure 3 a Log-Log plot of $k^{\prime \prime}$ as a function of $T$. The background attenuation noted $k_{\mathrm{B}}^{\prime \prime}$ on figure 2 has been deduced as follow ; first, we have chosen a variation of $k_{\mathrm{B}}^{\prime \prime}$ with temperature corresponding to the contribution of classical losses associated with the shear viscosity at $T<T_{\lambda}$ [5]; secondly, the amplitude of $k_{\mathrm{B}}^{\prime \prime}$ at $T=1.9 \mathrm{~K}$ has been adjusted to lead to a power law of the form $k_{\text {critical }}^{\prime \prime} \simeq(\varepsilon)^{-n}$. A variation of $10 \%$ for $k_{\mathrm{B}}^{\prime \prime}(1.9 \mathrm{~K})$ corresponds to a variation of $15 \%$ for $n$; this variation of $k_{\mathrm{B}}^{\prime \prime}(1.9 \mathrm{~K})$ is the biggest one compatible with the power law verified on a temperature range extending from $250 \mathrm{mK}$ to $10 \mathrm{mK}$ below $T_{\lambda} . T_{\lambda}$ is well known and is not a free adjustable parameter. The resulting line on figure 3 has a slope 0.65 ; the above discussed uncertainties on the background values $k_{\mathrm{B}}^{\prime \prime}$ give a rather bad accuracy on the critical exponent : $n=0.65 \pm 0.15$.

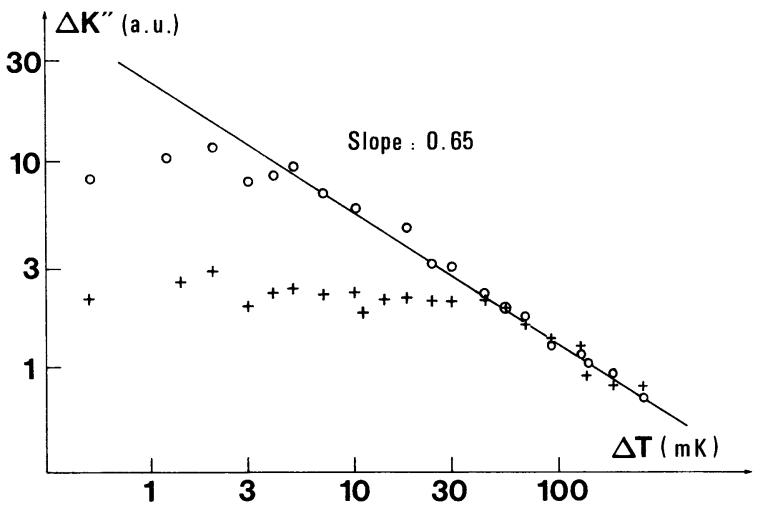

FIG. 3. - Log-Log plot of the critical ultrasonic attenuation versus $\Delta T=T_{\lambda}-T ;$ the straight line corresponds to a critical exponent 0.65 .

How is it possible to explain these results? The mechanism formely involved to explain the critical behaviour of $k^{\prime \prime}$ at low frequency no longer operates at our frequency because $\omega \tau_{2} \gg 1$ in our temperature range. It is therefore possible to suggest the following one. As in the case of the second sound, where the characteristic time is $\tau_{2} \simeq \xi / c_{2}$, in the case of the first sound, a characteristic time $\tau_{1}$ may be constructed

$$
\tau_{1} \simeq \frac{\xi}{c_{1}}
$$

$\tau_{1}^{-1}$ is the frequency of a phonon mode whose wavelength is of the order of the coherence length; it matches conditions required for strong interactions with critical modes. The existence of two characteristic frequencies $\tau_{2}^{-1}$ and $\tau_{1}^{-1}$ derived from the second and first sound has already been underlined by J. Swift and L. P. Kadanoff [10].

As long as the wave vector $k$ of the acoustical wave corresponds to

$$
k \xi<1
$$

we remain in the hydrodynamic regime and (6) is equivalent to $\omega \tau_{1}<1$.

It now becomes easy to use arguments based on the dynamical scaling hypothesis [1]. For this purpose we consider the normalized shape function of the correlation density :

$$
f_{\rho_{\mathrm{k}}}(x)=\frac{1}{\pi} \frac{\eta_{\mathrm{k}}}{\left(1-x^{2}\right)^{2}+\eta_{\mathrm{k}}^{2}}
$$

where

$$
x=\frac{\omega}{\omega_{\mathrm{k}}}, \quad \eta_{\mathrm{k}}=\frac{D_{1} k^{2}}{c_{1} k} .
$$

$\omega_{\mathrm{k}}=c_{1} k$ and $D_{1} k^{2}$ is the absorption per unit time of the acoustical wave. According to dynamical scaling $\eta_{k}$ depends on temperature only in the combination $k \xi$; this means that

$$
D_{1} \simeq b c_{1} \xi
$$

where $b$ is a constant of order unity.

This last result is equivalent to the assumption that the acoustical mode breaks up into two phonons modes [10] and it is therefore possible to find a single homogeneous function describing both the incident acoustic mode and the intermediate phonon modes. It corresponds to the simple diagramm :

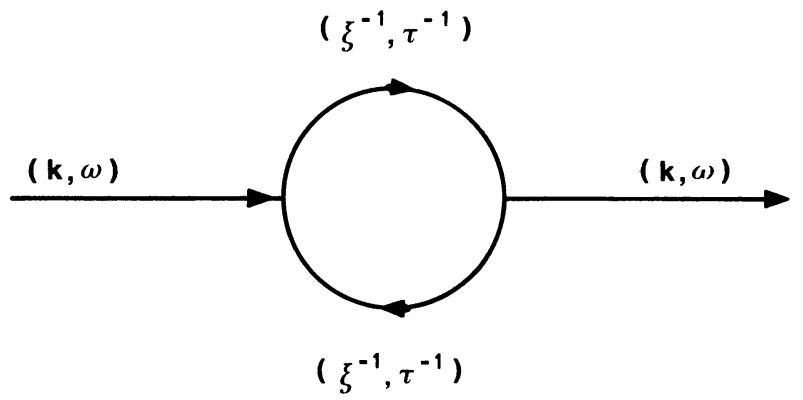

The resulting attenuation for this acoustical wave is :

$$
\alpha=\frac{\Delta c_{1,1}}{c_{1}^{2}} \frac{\omega^{2} \tau_{1}}{1+\omega^{2} \tau_{1}^{2}} .
$$

This implies that $\alpha \sim \omega^{2}$ and $\alpha \sim \varepsilon^{-2 / 3}$ near $T_{\lambda}$ as long as $\omega \tau_{1}<1$. This is exactly the divergence that we observe. $\Delta c_{1,1}$ is the difference in velocity at infinite frequency and at zero frequency and it is related to the anharmonic coupling of phonons (the vertex in the above diagram).

We now try to estimate its order of magnitude. In order to do this, we describe the anharmonicity of helium by a Grineisen constant defined by

$$
\frac{\mathrm{d} c_{1}}{\mathrm{~d} P}=\frac{\gamma}{\rho c_{1}}
$$

where $P$ is the pressure and $\rho$ the density of liquid helium; the experimental value [11] gives $\gamma \simeq 3$. Furthermore, the free energy of helium is described 
to a good approximation by the sum of two terms : $F_{0}$ depending only of the volume $V$ and $F_{1}$ resulting from the assembly of oscillators of frequencies

$$
\omega_{\mathrm{k}}=c_{1} k \text {. }
$$

$F_{1}$ is temperature dependent as a result of the condition for statistical equilibrium and volume dependent through $c_{1}$.

One can easily show that :

$$
\begin{aligned}
\frac{\Delta c_{1,1}}{c_{1}} & \simeq-\frac{1}{2 c_{1}^{2} \rho} V \frac{\partial^{2} F_{1}}{\partial V^{2}} \\
& \simeq\left(\frac{C}{V}\right) T \frac{1}{8 c_{1}^{2} \rho}\left(3 \gamma^{2}-\gamma\right)
\end{aligned}
$$

where $C / V$ is the $T^{3}$ component of the specific heat of helium (phonon specific heat only).

Using the calculated value

$$
\frac{C}{V}=23 \times 10^{4} \mathrm{erg} / \mathrm{cm}^{3} \mathrm{~K}
$$

at $2 \mathrm{~K}, \gamma \simeq 3$, we obtain

$$
\frac{\Delta c_{1,1}}{c_{1}} \simeq \frac{1}{50}
$$

The last result must be compared with the experimental value deduced from (10). If we suppose $\omega \tau_{1} \simeq 1$ for $\Delta T=T_{\lambda}-T_{1}$ where the attenuation is $2 \times 10^{5} \mathrm{~cm}^{-1}$, we find an experimental value

$$
\frac{\Delta c_{1,1}}{c_{1}} \simeq \frac{1}{10}
$$

of the same order of magnitude as the theoretical value.

Thus, two arguments justify our picture : the temperature dependence of the divergence of $k^{\prime \prime}$ and the order of magnitude.

The critical behaviour of the attenuation is described as well at high frequency with the characteristic time $\tau_{1}$ as it is at low frequency with the characteristic time $\tau_{2}$; the transition between the low frequency regime and the high frequency one would be, in our temperature range, between $100 \mathrm{MHz}$ and $1 \mathrm{GHz}$.

The last feature of our results to be explained corresponds to the different behaviour of $k_{1}^{\prime \prime}$ and $k_{2}^{\prime \prime}$ in the vicinity of $T_{\lambda}$. Two effects are possible $: k \xi$ becomes of the order of unity and consequently $k^{\prime \prime}$ saturates because the condition $\omega \tau_{1} \simeq 1$ is reached in the same temperature range. In fact, this would lead to the same behaviour for $k^{\prime \prime}$ measured with the first and second resonances. Since this does not occur we believe that such a difference is related to the onset of superfluidity conditions in the film. Such an effect has been treated by Ginzburg and Pitaevsky [12] and the result of the quenching of $\rho_{\mathrm{s}}$ on the surfaces of the film is that the temperature transition is lowered from $T_{\lambda}$ to $T_{\mathrm{d}}$. For $\rho_{\mathrm{s}}=0$ on the surfaces, it may be shown that

$$
\frac{T_{\lambda}-T_{\mathrm{d}}}{T_{\lambda}}=\left(\frac{\pi \xi_{0}}{d}\right)^{1 / \mu}
$$

with $\xi_{0}=1.45 \AA, v=0.67$, we compute

$$
\Delta T_{1}=45 \mathrm{mK}
$$

for $d=\lambda / 4=60 \AA$ and

$$
\Delta T_{2}=9 \mathrm{mK}
$$

for $d_{2}=\frac{3 \hat{\lambda}}{4}=180 \AA$.

The experimental results are in reasonable agreement with these calculated values. They are also in reasonable agreement with the onset temperature of superfluidity obtained from the third sound velocity measurements $[13,14]$ when they are extrapolated to our conditions. Of course this quenching not only modifies the temperature transition of the film but also, in an unknown fashion, the behaviour of the fluctuations in close vicinity of $\Delta T_{1}$ and $\Delta T_{2}$; this modification is difficult to evaluate with our results.

Finally we remark that for the first resonance we have at the cut-off $k \xi=0.5$, and for the second resonance $k \xi=1.5$. In fact when we approach the non hydrodynamic regime the damping time $\tau_{1}$ must be written

$$
\tau_{1}^{\prime} \simeq \tau_{1}\left(1+k^{2} \xi^{2}\right)^{-0.5} .
$$

The influence of $k \xi$ is then negligible for the first resonance but not for the second one. To sum up, we can say that the results are clear until $\Delta T \simeq 10 \mathrm{mK}$, but that closer to $T_{\mathrm{c}}$, several effects combine to render a quantitative interpretation of the experimental results more difficult.

In conclusion we emphasize that we have observed a critical attenuation for sound attenuation in liquid helium at a high frequency of $9 \mathrm{GHz}$ with a critical exponent $2 / 3$; it corresponds to an inhomogeneous decay into two phonons. The onset of superfluidity has also been observed for rather thick films. 


\section{References}

[1] Hohenberg, P. C., Proceedings of the International School of Physics Enrico Fermi, course 51 (1971) 285.

[2] Khalatnikov, I. M., Zh. Eksp. Teor. Fiz. 57 (1969) 489 [Sov. Phys. JETP 30 (1970) 268].

[3] Williams, R. D. and Rudnick, I., Phys. Rev. Lett. 25 (1970) 276.

[4] Ahlers, G., J. Low Temp. Phys. 1 (1969) 609.

[5] Imai, J. S. and Rudnick, I., Phys. Rev. Lett. 22 (1969) 694.

Commins, D. E. and Rudnick, I., Proc. 13th Int. Conf. Low Temp. Phys. 1 (Plenum Press, New York) 1974, p. 532.

[6] Frenois, Ch., Joffrin, J., Legros, P. and Levelut, A., Phys. Rev. Lett. 32 (1974) 1295.
[7] Sabisky, E. S. and Anderson, C. H., Phys. Rev. 7 (1973) 790.

[8] Barmatz, M. and Rudnick, I., Phys. Rev. 170 (1968) 224.

[9] Berberich, P., Leiderer, P. and Hunklinger, S., J. Low Temp. Phys. 22 (1976) 61.

[10] Swift, J. and Kadanoff, L. P., Ann. Phys. 50 (1968) 312.

[11] Abraham, B. M., Eckstein, Y., Ketterson, J. B., Kuchnir, M. and Roach, P. R., Phys. Rev. A 1 (1970) 250.

[12] Ginsburg, V. L. and Pitaevsky, L. P., Zh. Eksp. Teor. Fiz. 34 (1958) 1240 [Sov. Phys. JETP 7 (1958) 858].

[13] Kagiwada, R. S., Fraser, J. C., Rudnick, I. and Bergman, D., Phys. Rev. Lett. 22 (1968) 338.

[14] Scholtz, J., Mac Lean, E. and Rudnick, I., Phys. Rev. Lett. 32 (1974) 147. 